\title{
Experimental Demonstration of Plasma Lens Focusing
}

\author{
G. Hairapetian, P. Davis, C. E. Clayton, C. Joshi \\ Department of Electrical Engineering, UCLA, 405 Hilgard Avenue, Los Angeles, CA 90024 USA \\ S. Hartman, C. Pellegrini \\ Department of Physics, UCLA, 405 Hilgard Avenue, Los Angeles, CA 90024 USA \\ T. Katsouleas \\ Department of Electrical Engineering, USC, Los Angeles, CA 90089 USA
}

\begin{abstract}
The magnetic self-focusing of a relativistic electron beam propagating through a plasma is demonstrated. The plasma which is produced by an RF discharge in a glass tube with no externally applied magnetic field, focuses a $3.5 \mathrm{MeV}, 25 \mathrm{ps}$ (FWHM) long electron beam from an initial size of $2.5 \mathrm{~mm}$ (FWHM) to about $0.5 \mathrm{~mm}$ (FWHM) at a focal length of 18 $\mathrm{cm}$.
\end{abstract}

\section{INTRODUCTION}

A relativistic electron beam propagating through a plasma can self pinch due to its self generated azimuthal magnetic field[1]. In vacuum, the beam generated Lorentz force counteracts the radial space charge force and the beam propagates with an equilibrium radius. As the beam enters the plasma, the plasma electrons re-distribute themselves to charge neutralize the beam. If the beam radius is small ( $r<$ $c / \omega_{p}=$ collisionless skin depth), most of the plasma return currents will flow outside of the beam. As a result, the beam magnetic field will not be reduced appreciably within the beam, and it will focus under its own radial Lorentz force. This mechanism can be used to generate focusing gradients exceeding MG $/ \mathrm{cm}$ which is several orders of magnitude greater than the strength of conventional quadrupole magnets[2].

While a large number of theoretical studies[2],[3] of this effect have been performed, there have been only two experimental studies[4],[5]. In the first experiment[4], the plasma length $(35 \mathrm{~cm})$ was much longer than the focal length
$(8 \mathrm{~cm})$, and the spot size was measured outside of the plasma column. In the second experiment[5], the beam focused outside of the plasma column but the amount of radial focusing was very limited (in some cases the spot size variations were within the experimental errors ). In this paper, we report on a plasma lens experiment at UCLA, where a 3.5 $\mathrm{MeV}$ electron beam was focused from an initial spot size of $2.5 \mathrm{~mm}$ (FWHM) to ahout $0.5 \mathrm{~mm}$, well outside of the plasma column.

\section{EXPERIMENTAL SETUP}

The experiment was performed with the UCLA's 4.5 $\mathrm{MeV}$, laser driven RF gun[6]. The gun is capable of producing very short ( $\tau<10 \mathrm{ps}$ ) electron bunches $\left(\mathrm{Q}>1 \mathrm{nC}, \mathrm{I}_{\text {peak }}>\right.$ $100 \mathrm{~A})$ at $1 \mathrm{~Hz}$. In order to simplify the diagnostics, the electron bunches were stretched to about 25 ps (FWHM) by illuminating the cathode with a longer laser pulse. The photo electrons are accelerated to about $3.5 \mathrm{Mev}$ in the 1.5 cell RF gun and transported using a focusing solenoid and four steering magnets to the plasma chamber $2 \mathrm{~m}$ downstream of the gun (Figure 1). The plasma chamber which is filled with Ar gas up to a pressure of $30 \mathrm{mT}$ Torr is connected to the RF gun ( $\mathrm{p}<5 \times 10^{-8}$ Torr) through a windowless, two stage differential pumping system (Figure 1). Each pumping stage consists of a low conductance tube followed by a turbomolecular pump. The first stage maintains a pressure differential of three orders of magnitude; while the second, a differential of two orders of magnitude. The electron beam which initially contains up to $1.5 \mathrm{nC}$ of charge, is scraped by

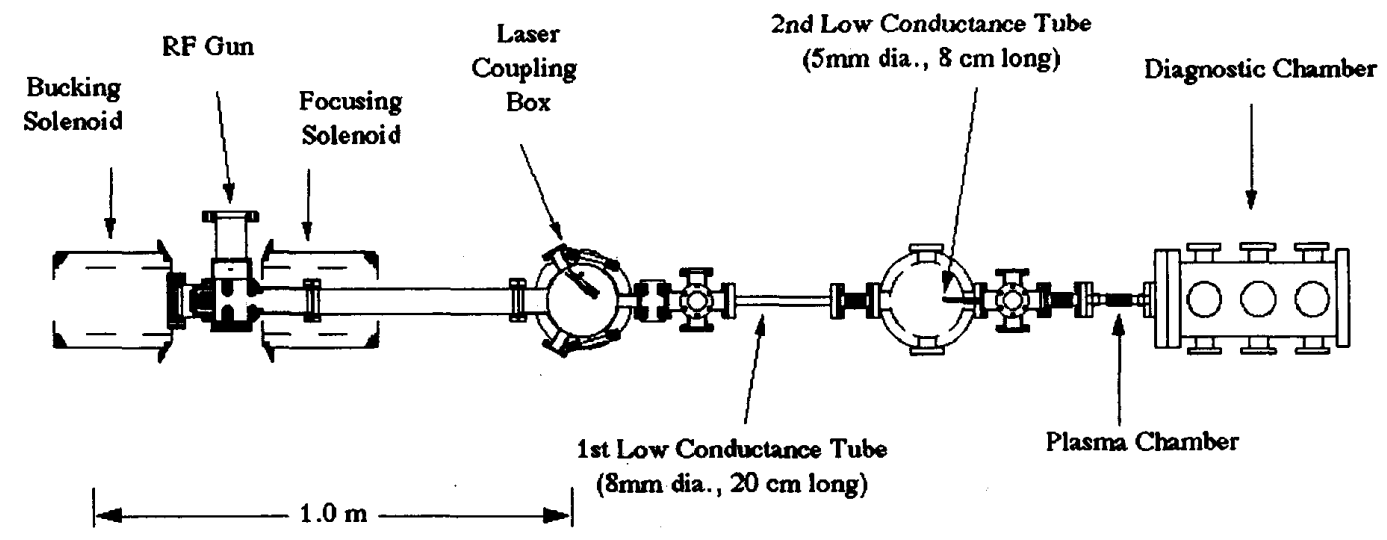

Figure 1. Experimental Setup 
the two low conductance tubes, and a maximum charge of 0.6 $\mathrm{nC}$ is transported to the plasma chamber. Beam diagnostics include: phosphor screens both upstream and downstream of the plasma chamber, retractable faraday cups, a current transformer for non-destructive measurement of beam charge, and a Cerenkov radiator downstream of the plasma chamber.

The plasma is produced by an RF discharge in a glass tube $($ diam. $=1.7 \mathrm{~cm}$, length $=10 \mathrm{~cm})$ with no external magnetic field. The RF amplifier $(10-20 \mathrm{MHz}, 800 \mathrm{~W})$ is connected via a capacitive tuning circuit to a helical antenna wrapped on the outside of the glass tube filled with Argon gas. The helical antenna ( $5 \mathrm{~cm}$ long, 12 turns) induces large azimuthal electric fields inside the tube where the Ar gas is ionized by impact ionization. Under optimum tuning, about $75 \%$ of the input RF power is coupled to the plasma. The RF plasma density is diagnosed by a small cylindrical (diam. = $0.76 \mathrm{~mm}$, length $=2.5 \mathrm{~mm}$ ) Langmuir probe. Figures $2(\mathrm{a})$ and (b) show typical radial and axial density profiles. The radial profile, measured at an axial position $0.5 \mathrm{~cm}$ away from the edge of the antenna, has a flat profile in the middle and decreases sharply near the tube walll. Axially, the plasma density peaks under the antenna and falls rapidly away from

a)

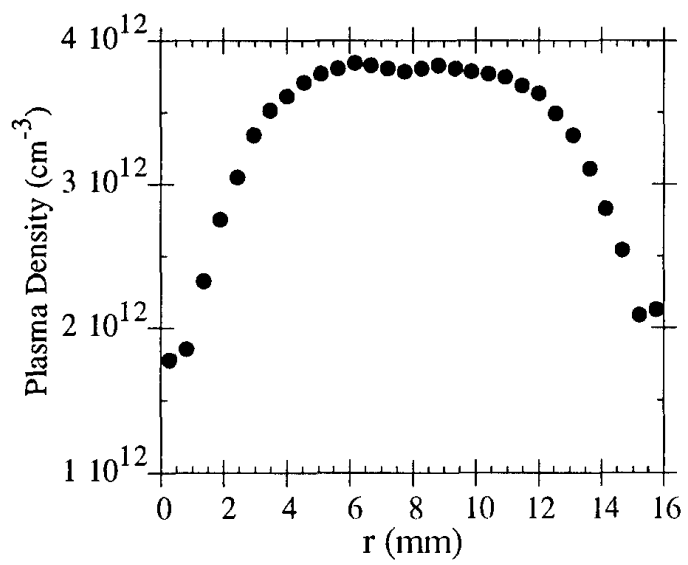

(b)

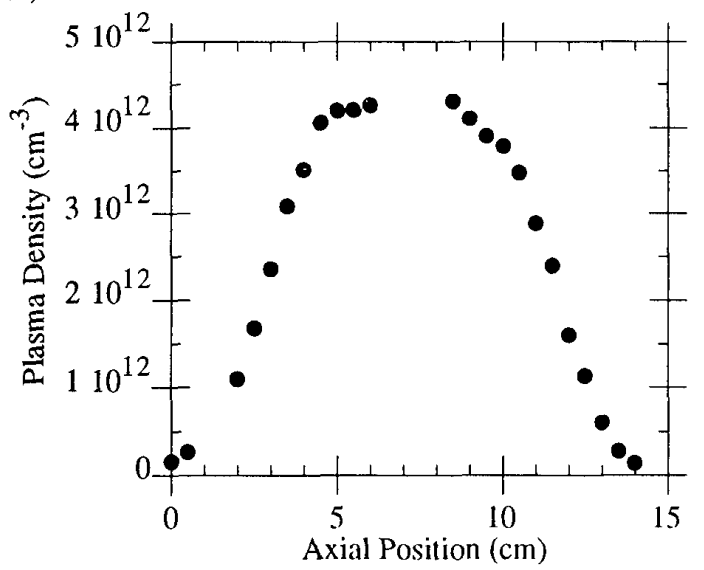

Figure 2. (a) Radial profile of plasma density. (b) Axial profile of plasma density. the antenna $(\mathrm{n} / \nabla \mathrm{n} \cong 5 \mathrm{~cm})$. The density directly underneath the antenna cannot be measured because the Langmuir probe perturbs the RF discharge. The plasma density can be varied over an order of magnitude by varying the gas pressure and the RF power.

The plasma density has to be high enough to charge neutralize the beam in a time less than the electron bunch length $\tau=25 \mathrm{ps}$; i.e. $\tau \omega_{\mathrm{p}}>1$ where $\omega_{\mathrm{p}}=\left(4 \pi n \mathrm{e}^{2} / \mathrm{m}\right)^{1 / 2}$. Hence, the minimum required plasma density is $n=5 \times 10^{11}$ $\mathrm{cm}^{-3}$ which can easily be attained by this source. The transverse beam size is about $3 \mathrm{~mm}$ FWHM $\left(<\mathrm{c} / \omega_{\mathrm{p}}\right)$ at the entrance of plasma which implies that the beam density is approximately $n_{b}<4 \times 10^{10} \mathrm{~cm}^{-3}\left(n_{b}<n_{\text {plasma }}\right)$; i.e., the lens operates in "overdense" plasma regime. One can roughly estimate the focal length of the lens from the "thin" lens approximation, which is given by[2]

$$
\mathrm{f}=\left(\mathrm{c} / \omega_{\mathrm{bp}}\right)^{2}(2 \gamma / \mathrm{l})
$$

where $f$ is the focal length, $l$ is the plasma length, $c$ is speed of light, and $\omega_{b p}$ is the beam plasma angular frequency. For a 5 $\mathrm{cm}$ long plasma and a typical beam density of $3 \times 10^{10} \mathrm{~cm}^{-3}$, the predicted focal length is about $27 \mathrm{~cm}$. The focal length

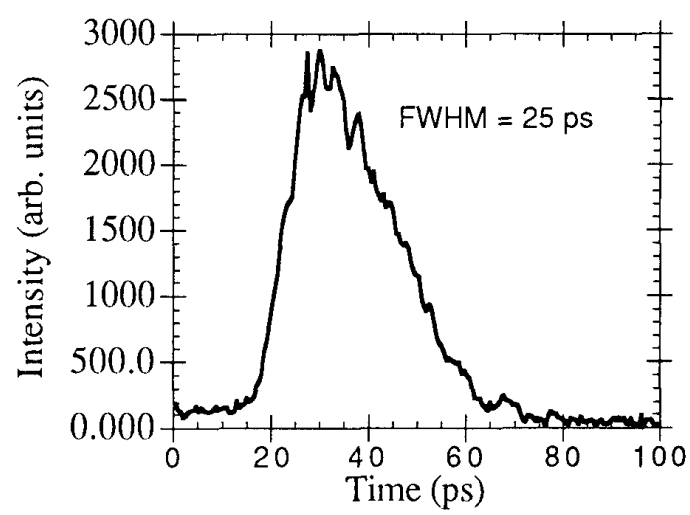

Figure 3. Temporal Profile of the electron beam.

calculation is for a beam with uniform density in space and time. In reality, the focal strength of the lens is not constant and has both longitudinal and radial variations.

\section{EXPERIMENTAL RESULTS}

The time integrated electron beam size and the total charge were measured $22 \mathrm{~cm}$ upstream of the plasma. The beam is about $2.5 \mathrm{~mm}$ FWHM in the vertical dimension, and contains up to $.6 \mathrm{nC}$ of charge. The electron bunch length is measured $27 \mathrm{~cm}$ downstream of plasma source by streaking the Cerenkov ligh from a $0.5 \mathrm{~mm}$ thick fused silica[7]. Figure 3 shows the temporal profile of the electron bunch. It has a sharp rise and a fairly long tail. The electron beam propagates through the plasma and hits the first phosphor screen $18 \mathrm{~cm}$ downstream of the plasma. Figure 4(a) shows the time integrated beam size at the first phosphor screen with no 

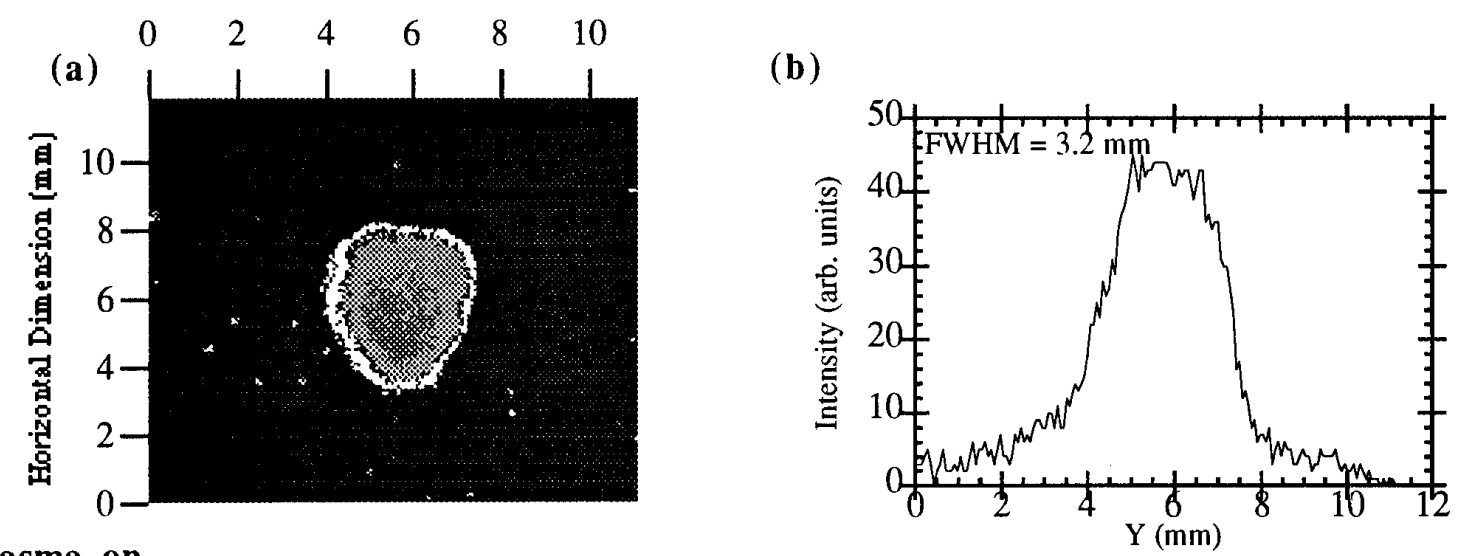

\section{Plasma on}
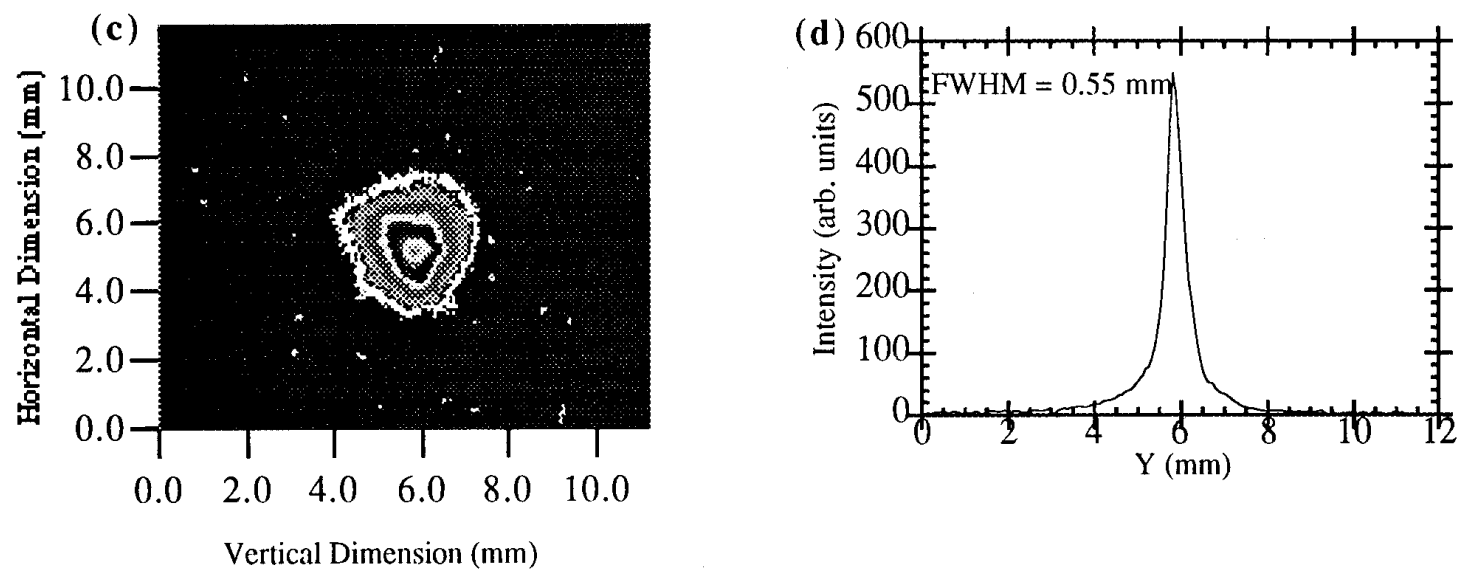

Figure 3. (a) Unfocused beam image $18 \mathrm{~cm}$ downstream of plasma. source. (b) Vertical line out of image (a). (c) Focused beam image. (d) Vertical line out of image (c).

plasma. The beam dimensions are measured by taking either a vertical or horizontal line-out through the pixel with the highest intensity. As Figure 4 (b) shows, the vertical dimension of the unfocused beam is about $3.2 \mathrm{~mm}$ FWHM. When the plasma is turned on, the beam focuses down to about $0.55 \mathrm{~mm}$ (Figs. 4 (c) and (d)). The spot size increases to about $1.1 \mathrm{~mm}$ at a second phosphor screen, $22 \mathrm{~cm}$ downstream of the first. The apparent plasma focal length (18 $\mathrm{cm}$ ) is shorter than that predicted from the thin lens approximation $(27 \mathrm{~cm})$. This can be attributed in part to the finite length of the plasma. As the beam propagates through the plasma, it begins to focus within the lens, increases its density, and shortens the lens focal length.

\section{FUTURE WORK}

To learn more about the time dependent focusing of the beam, the Cerenkov radiation from the focused electron beam has been streaked using a fast streak camera (temporal resolution $=3.5 \mathrm{ps}$ ). Currently, we are in the process of analyzing these results which will be reported elsewhere. We also plan to investigate the effects of beam and plasma density on the focusing. Furthermore, experimental results will be compared with both particle simulation and analytical calculation results.

\section{ACKNOWLEDGMENTS}

The authors acknowledge helpful discussions with J.B. Rosenzweig, J.J. Su, and H. Zwi. Also, expert technical assistance from N. Barov, M. Fauver, M. Hogan, P. Kwok, S. Park, G. Travish, and R. Zhang is greatly appreciated. This work is supported by SDIO/IST through ONR Grant N0001490-J-1952 and US DOE Grant DE-FG03-92ER-40493.

\section{REFERENCES}

[1] P. Chen, Part. Accel. 20, 171 (1987).

[2] J.J. Su et. al.., Phys. Rev. A 41, 3321 (1990), and References therein.

[3]P. Chen, Phys. Rev. A 45, r3398 (1992).

[4] J. B. Rosenzwieg et. al., Phys. Fluids B 2 (6), 1376 (1990).

[5] H. Nakanishi et. al., Phys. Rev. Lett. 66, 1870 (1991).

[6] C. Pellegrini et. al., these proceedings.

[7] G. Hairapetian et. al., these proceedings. 


\title{
Numerical Modelling of Time-Space Behavior of High-Current Relativistic Electron Beam in Plasma Waveguide
}

\author{
Vyacheslav I.Karas' \\ Kharkov Institute of Physics and Technology Ukrainian Academy of Sciences \\ 310108 Akademicheskaya Str. 1, Kharkov-108, Ukraine \\ Nadya G.Belova \\ Institute of Physics and Technology Russian Academy of Sciences \\ 117218 Krasikov Str. 25a, Moscow, Russia
}

\begin{abstract}
The physical processes in the magnetized plasma waveguide, in which the high-current relativistic electron beam (Sudan parameter $S>1$ ) is injected, are investigated by the developed 2.5-D relativistic electromagnetic code. The computer study has shown that the charge and current compensations of the high-current beam are considerably different from those of the low-current beam. In addition the self-consistent electromagnetic fields generated by a beam at the initial state radically alter both the linear and the nonlinear instability stages.
\end{abstract}

\section{INTRODUCTION}

The advantages of a use of the high-current relativistic electron beam (HCREB) for development of a powerful electromagnetic radiation sources, and the new type accelerators based on the collective methods of the particles acceleration, and etc. are presented in [1]-[3]. It was noted that the simultaneous growth both of the beam energy and the non-equilibrium degree of a system defining by the Sudan parameter $S=\left(n_{b} / n_{e}\right)^{1 / 3} \gamma\left(n_{b}, n_{e}\right.$ are the beam and plasma densities, $\gamma$ is the relativistic factor) is a very important fact. In the unbounded systems this can results in the decrease of the energy (at $S>1$ ) transferring from a beam to the plasma for the oscillations excitation. In the system bounded in the radial direction this fact decreases the efficiency of the electromagnetic waves radiation due to the oscillations excitation with the small ratio between the transverse and longitudinal components $[3,4]$. Cerenkov mechanism was proved to be changed to the anomalous Doppler mechanism for HCREB [5]. This allows to remain the high efficiency of the electromagnetic radiation at the certain parameters of a system such as the magnetized plasma waveguide (MPW) - HCREB.

The equilibrium and stability conditions of HCREB were investigated in many works (see Refs. in [1]-[5]). The injection of the low-current $(S \ll 1)$ stringent REB is also studied in detail both with the magnetic field and without field. It was shown if the beam radius $a$ to be greater than the skindepth $\lambda_{E}=c / \omega_{e}$ ( $c$ is the light velocity, $\omega_{e}$ is the Langmuir plasma frequency) the beam current is compensated by a return plasma current damping in a plasma with the finite conductivity. The external magnetic field modifies the current compensation condition to the form $a \gg \lambda_{E}\left(1+\Omega_{e}^{2} / \omega_{e}^{2}\right)$ ( $\Omega_{e}$ is the Larmor electron frequency).

In this work both the charge-current compensation and the stability of HCREB $(S>1)$ in MPW are investigated. The hollow narrow beam of the $a$ radius and of the $\Delta_{b}$ thick- ness (so that $\Delta_{b}<c / \omega_{e}$, but $a>c / \omega_{e}$ ) is considered, and the hollow wide beam with $\Delta_{b} \approx r_{L}\left(r_{L}\right.$ is the waveguide radius) is also. In the both cases the Cerenkov resonance condition of the beam with the plasma wave is not valid i.e. $\gamma>\omega_{e} /\left(c k_{\perp}\right)\left(k_{\perp}\right.$ is a transverse wave number). For comparison, the study of the low-current beam is also presented.

\section{MODEL AND EQUATIONS}

In order to study the dynamics of a collisionless plasma with the relativistic electron beam in both the self-consistent and the external electromagnetic fields in axisymmetric $(\partial / \partial \theta=0)$ geometry, we use the set of relativistic Vlasov's equations for the distribution functions of the given type of particles $f_{s}(\vec{p}, \vec{R}, t)$. Here $\vec{p}=m_{s} \vec{v} \gamma, \vec{v}=\{\dot{r}, r \dot{\theta}, \dot{z}\}$, $\gamma=\left[1-(|\vec{v}| / c)^{2}\right]^{-1 / 2}$ is the relativistic factor, $\vec{R}=\{r, z\}$. The self-consistent electromagnetic fields in Vlasov's equation are determined by Maxwell's equations in the form of wave equations for the dimensionless scalar $\phi$ and vector $\overrightarrow{4}$ potentials in which the right side is defined by the total charge and current densities [6].

We will consider the infinite value of the uniform external magnetic field $H \rightarrow \infty$ then the motion of the particles can be treated as the one-dimensional. Thus we have the only equation of the motion and the equations for the potentials $\phi(r, z)$ and $A_{z}(r, z)$

In these equations the quantities involved are used in the dimensionless form: $[v]=c ;[r, z]=c / \omega_{e} ;[t]=\omega_{e}^{-i}$; $[n]=n_{0 e} ;[q]=e ;[m]=m_{0} ;[\phi, A]=\mathcal{E}_{c h} / e_{i}[E, B]=$ $\left(4 \pi n_{0 e} \mathcal{E}_{c h}\right)^{1 / 2} ;[J]=e n_{0 e} c$, where $\omega_{e}=\left(4 \pi n_{0 e} e^{2} / m_{0}\right)^{1 / 2}$ is the electron plasma frequency, $\mathcal{E}_{c h}=m_{0} c^{2}$ is the rest energy of the beam electron, $n_{0 e}, m_{0}, e$ are the initial density, rest mass and charge of the electrons respectively.

The dimensionless equation of motion, obtained as characteristic equation of Vlasov's equation, was written as

$$
\frac{d u_{z}}{d t}=-\frac{q}{m}\left(\frac{\partial A_{z}}{\partial t}+\frac{\partial \phi}{\partial z}\right)
$$

where $u_{z}=\gamma v_{z}, \gamma=\left[1+u_{z}^{2}\right]^{1 / 2}$.

The boundary conditions for the potentials are

$$
\begin{aligned}
& r=0: \quad \partial \phi / \partial r=\partial A_{z} / \partial r=0 ; \\
& r=r_{L}: \quad \phi=A_{z}=0 \text {; } \\
& \left.\begin{array}{l}
z=0 \\
z=z_{L}
\end{array}\right\}, \phi=\frac{\partial A_{z}}{\partial z}=0
\end{aligned}
$$

The initial conditions for the self-consistent fields are $\Delta \phi=-\rho, A_{z}=0$. Here $\Delta$ is Laplassian. 